\title{
A CONTRIBUIÇÃO DA SBQ À PÓS-GRADUAÇÃo EM QUÍMICA
}

\author{
Solange Cadore* \\ Instituto de Química, Universidade Estadual de Campinas, CP 6154, 13084-971 Campinas - SP, Brasil \\ Jailson B. de Andrade \\ Instituto de Química, Universidade Federal da Bahia, Campus Universitário de Ondina, 40170-290 Salvador - BA, Brasil
}

\begin{abstract}
THE CONTRIBUTIONS OF SBQ FOR THE GRADUATE PROGRAMS IN CHEMISTRY. Brazilian Chemical Society (SBQ) enjoys 30 years dedicating to the development, consolidation and divulgation of research in the Chemistry area. There are graduate programs in chemistry spread through all Brazilian regions and different actions related to them have been supported by SBQ since its foundation. The chemistry divisions, the Annual Meeting, the journals Quimica Nova, JBCS and QNEsc as well as the Graduate Forum offer an opportunity to discuss and to divulge Chemistry in the present context but also its evolution considering the men and the environmental needs leading to the development of new methodologies for different parts of the society. This manuscript describes the importance and the contribution of SBQ to the development of graduate curses of Chemistry in Brazil
\end{abstract}

Keywords: graduate programs in chemistry; research in Brazil; chemistry in Brazil.

Pensar uma estratégia para o desenvolvimento científico levou à constatação, na metade do século passado, de que era necessário investir na formação de recursos humanos qualificados para atuar nas Instituições de Ensino Superior do país. Para isso, a Coordenação de Aperfeiçoamento de Pessoal de Nível Superior, CAPES, foi criada em 1951. A iniciativa bem sucedida, acompanhada de outras ações governamentais, como a criação do Conselho Nacional de Desenvolvimento Científico e Tecnológico, CNPq, e da Financiadora de Estudos e Projetos, FINEP, permitiu a criação e a expansão do sistema de Pós-Graduação no Brasil.

A Química, como ciência bem estabelecida, já trilhava o caminho da pesquisa em diferentes locais do Brasil, porém constituíam iniciativas isoladas de alguns cientistas perseverantes de nossa área e, geralmente, associadas a Faculdades de Filosofia, Ciências e Letras. Se em condições nem sempre favoráveis a pesquisa florescia, com o estímulo da CAPES abriu-se um novo horizonte para a área de Química. Em 1963, foi criado o Programa de Pós-Graduação em Química Orgânica da Universidade Federal do Rio de Janeiro e, a partir de então, outros Programas de Pós-Graduação em Química foram criados e, com o passar do tempo consolidaram-se, passando a formar recursos humanos qualificados que, por sua vez, multiplicaram esta iniciativa.

Durante algum tempo, havia poucos Cursos de Doutorado no país e, talvez por isso, criou-se a cultura da elaboração de Dissertações de Mestrado (na época, chamadas de teses de Mestrado, uma vez que, de fato, eram teses) com um alto nível, a qual foi difícil tentar adaptar à realidade mais recente, quando o tempo máximo de bolsa passou a ser de 24 meses. Esta forma de atuação levou ao desenvolvimento de uma Pós-Graduação marcada pela qualidade, acima de tudo, e esse padrão também foi levado para os Cursos de Doutorado, à medida que foram sendo criados.

Após a criação e a expansão da Pós-Graduação, a próxima etapa consistiu na necessidade de um sistema de avaliação, de forma a garantir que a qualidade desejada estivesse presente em todos os Programas. Também nesse aspecto a CAPES foi vitoriosa, de tal sorte que o Brasil tem, hoje, um dos melhores sistemas de avaliação da pós-graduação, servindo de exemplo para outros países.

Exemplo também foi e é o papel da Sociedade Brasileira de

*e-mail: cadore@iqm.unicamp.br
Química, criada há 30 anos, empenhando-se em congregar os Químicos, divulgar a Química, diagnosticar tendências, catalisar ações para o desenvolvimento científico e tecnológico da área e acompanhar o processo de ampliação, consolidação e avaliação da PósGraduação em Química.

A publicação de Química Nova permitiu que a pesquisa desenvolvida no Brasil pudesse ser divulgada de forma mais acessível, na língua pátria. A partir de janeiro de 1978, a SBQ passou a difundir a Química de forma irreversível e sempre crescente, o que pode ser constatado pela periodicidade da Revista, pelo número de artigos em cada revista, pelo tempo de publicação dos artigos aceitos, exigindo números extras para dar vazão ao grande número de trabalhos submetidos e aceitos após um trabalho criterioso de avaliação. Esta evolução mostra também a qualidade dos trabalhos publicados, o fator de impacto deste periódico, superior a muitos publicados em língua inglesa e, portanto, com maior difusão no cenário internacional ${ }^{1}$.

Uma parcela significativa da pesquisa desenvolvida nos diferentes Programas de Pós-Graduação é publicada em Química Nova. Além disso, periodicamente são divulgados trabalhos que mostram as tendências e perspectivas das diferentes sub-áreas da Química ${ }^{2-8}$.

Entretanto, antes de submeter seus trabalhos para Química Nova, os pós-graduandos os apresentam na Reunião Anual da SBQ. Após a realização da sua $30^{\mathrm{a}}$ edição, quem de nós nunca apresentou um trabalho na RABSQ? Poucos, certamente, uma vez que esse evento congrega toda a comunidade, das diferentes sub-áreas da Química, agora reunidas em Divisões. A possibilidade de apresentar seu trabalho, discutí-lo com outros pesquisadores e assistir às atividades programadas fazem parte do processo de formação dos alunos de Pós-Graduação. Muito do espírito crítico que tanto cobramos de nossos alunos começa e se aprimora nessas Reuniões.

A criação do Journal of the Brazilian Chemical Society confirma a vocação da SBQ como maior contribuinte para o desenvolvimento do ensino e pesquisa em nosso país. Este periódico, publicado em língua inglesa, permitiu uma maior abrangência de divulgação e também é responsável por boa parcela dos artigos publicados pelos Programas de Pós-Graduação.

Paralelamente à divulgação, a SBQ desde sua criação atua também em outras esferas, buscando garantir a consolidação da pesquisa em Química. Isto foi possível graças ao esforço e dedicação de Diretoria e Conselho, de todas as suas gestões, e de sócios da SBQ 
que atuaram e atuam como membros de comitê assessor, de conselho e como representante de área, etc. nos diferentes órgãos gestores da Educação e da Ciência e Tecnologia, responsáveis por editais de financiamento, bolsas de pesquisa, bolsas de pós-graduação, etc.

Assim, desde 1981 é possível encontrar textos em Química Nova mapeando o universo da pesquisa no Brasil e a distribuição de trabalhos publicados pelos pesquisadores das diferentes sub-áreas, descrevendo a atuação do CNPq e suas ações voltadas para o desenvolvimento da Ciência e Tecnologia, alertando para o problema de bolsas de Pós-graduação, ferramenta absolutamente necessária para o bom andamento das atividades, uma vez que permite ao aluno concentrar-se em seus estudos, sem ter que buscar outras formas para a sua sobrevivência ${ }^{2,9-13}$. Ao ler esses textos, infelizmente, muitas vezes só podemos definir quando ele foi escrito pela data da publicação, uma vez que a realidade descrita ainda é atual. Isto reforça a necessidade que a comunidade tem de estar sempre representada nos diferentes comitês e da importância desses registros escritos.

A Química é uma das poucas, senão a única área que tem o registro de todas as avaliações dos Programas de Pós-Graduação ${ }^{14-}$

${ }^{16}$. Com o passar dos anos diferentes indicadores foram sendo introduzidos no processo de avaliação, até chegar aos dias atuais. Este sistema de avaliação pode não agradar a todos, mas constitui um balizamento para o aperfeiçoamento e a consolidação dos Programas. Mais do que isto, é importante ressaltar que os Programas participaram do processo que levou ao atual estágio de avaliação, pela atuação da SBQ.

$\mathrm{O}$ crescimento do número dos Programas exigiu que os mesmos se unissem no sentido de lutar por objetivos comuns. Durante alguns anos, entretanto, foram pouco frequientes as reuniões entre o Representante da área de Química e os Coordenadores dos Cursos de Pós-Graduação em Química.

A primeira dessas reuniões foi realizada em São Carlos, SP, em 1991, organizada pelo professor Timothy J. Brockson, na época o Representante de área junto à CAPES. Além de reunir os Coordenadores dos Programas com o representante da Capes, o objetivo deste encontro foi fazer uma análise da Pós-Graduação em Química no País. Em 1993, durante a Assembléia Geral da 16a Reunião Anual da Sociedade Brasileira de Química foi constituída uma comissão composta pelos professores Jailson Bittencourt de Andrade (UFBA), Heloisa Helena Ribeiro Schor (UFMG), Marco Antonio Chaer do Nascimento (UFRJ), Timothy J. Brocksom (UFSCAR/ CAPES), Faruk José Nome Aguilera (UFSC) e Eliezer J. Barreiro (UFRJ) e pelo então discente de pós-graduação, Marcelo Giordan (UNICAMP), para dar continuidade aos trabalhos iniciados em 1991. Esta comissão, após intensas discussões, concluiu que medidas diversas deveriam ser tomadas no sentido de fortalecer a Pósgraduação em Química no País: investimento para a infra-estrutura instrumental dos Programas; fortalecimento do sistema de bibliotecas; capacitação de estudantes para o magistério; definição da filosofia a ser utilizada na formação de mestres e doutores ${ }^{17}$.

A partir dessas discussões a SBQ elaborou um documento, o qual foi apresentado ao MCT, ao CNPq e à Comissão de C\&T da Câmara dos Deputados ${ }^{18}$.

Em agosto de 1996, a Sociedade Brasileira de Química promoveu um encontro em Salvador, BA, onde reuniram-se os Coordenadores de Pós-Graduação, representantes da SBQ, da CAPES e do CNPq. Mais uma vez Química Nova foi o veículo de divulgação dos resultados desse evento ${ }^{14}$.

Em maio de 2002, foi organizado, sob a coordenação da Professora Solange Cadore (UNICAMP) o workshop "Um Olhar Sobre a Pós-Graduação em Química no Brasil”, como parte das comemorações dos 25 anos da Sociedade Brasileira de Química. Este evento contou com a presença de Coordenadores de Programas de Pós-
Graduação, dos representantes de agências de fomento, $(\mathrm{CNPq}$ e CAPES), além do Representante da área de Química. A sistemática de avaliação dos Programas e os critérios utilizados na avaliação pela CAPES foram bastante discutidos, assim como a situação das Agências CNPq e CAPES com relação ao fomento às atividades de pós-graduação, destacando as bolsas de formação e o papel que os Fundos Setoriais podem desempenhar na expansão do sistema. Por fím, ficou decidido que após a divulgação dos resultados da Avaliação Continuada (segundo semestre de 2002), deveria ser organizada uma nova reunião envolvendo a SBQ, os Coordenadores de Programas de PG em Química e o CA/CAPES, para apreciar o desempenho da área e propor, se necessário, novos rumos.

Assim, foi realizado o workshop "A Química no Brasil: Situação Atual e Perspectivas" em dezembro de 2002, em Salvador, BA, cujo objetivo principal foi dar continuidade às discussões sobre o Ensino, Pesquisa e Avaliação em Química, com a participação de Coordenadores de Programas de Pós-Graduação em Química, Diretoria e Conselho da SBQ e Membros dos Comitês Assessores de Química na CAPES, CNPq e Fundações Estaduais de Fomento. Os assuntos discutidos e as deliberações da reunião fizeram parte do documento "Eixos Mobilizadores da Química Brasileira", que foi apresentado ao governo brasileiro e encontra-se publicado ${ }^{19}$.

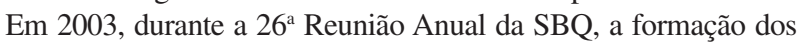
profissionais de Química, sua absorção pelo setor industrial e os reflexos desses profissionais no ensino foram discutidos no simpósio “A Formação do Químico". Os Programas de Pós-Graduação em Química são uma fonte de novos profissionais, altamente qualificados, que podem atuar em ambos os sistemas, acadêmico e industrial. Para discutir com maior profundidade o perfil do profissional em Química e o papel da pós-graduação no Ensino, na Pesquisa e na Avaliação em Química, foi o realizado o $3^{\circ}$ Workshop de Pós-Graduação em Química, com o tema "O Papel da Pós-Graduação na Formação do Químico". As discussões deste evento resultaram num documento que foi publicado ${ }^{20}$ e que serviu de base para discussões entre a SBQ e o Centro de Gestão de Estudos Estratégicos, CGEE.

A importância dos Programas de Pós-Graduação na formação do Químico e no futuro desses profissionais, sua absorção pelas empresas, bem como a transferência de tecnologia desenvolvida nas Universidades, para o setor não-acadêmico, e os desafios futuros da Pós-Graduação foram temas das últimas edições do Workshop.

Em 2005, durante o $4^{\circ}$ Workshop, foi criado o Fórum de PósGraduação, responsável pela discussão de questões de interesse dos diferentes Programas.

É inegável que a Pós-Graduação em Química está evoluindo, em quantidade e em qualidade. $\mathrm{O}$ número de pós-graduandos cresce e, conseqüentemente, também cresce o número de Mestres e de Doutores (Figura 1) e a produção de artigos científicos (Figura 2). Este crescimento torna-se exponencial na década de 1990 e um dos aceleradores foi o Programa de Apoio ao Desenvolvimento Científico e Tecnológico, PADCT ${ }^{22,23}$. Assim, a cada ano aumenta o contingente de doutores, que são pesquisadores qualificados para entrar no mercado de trabalho, que consiste, na maior parte dos casos, das Instituições de Ensino Superior ${ }^{24}$.

Como esses novos doutores nem sempre podem ser absorvidos pelos Programas já estabelecidos e consolidados, unem-se a outros jovens pesquisadores e começam a nuclear grupos de pesquisa em novos centros. Em pouco tempo, surge a necessidade de criar novos Programas. Desta forma, apesar da recomendação da CAPES, de unir diferentes Cursos de um mesmo Programa, o número de Programas de Química credenciados passou de 42, em 2004, para 61 em 2007, assim discriminados: 23 Cursos de Mestrado, 1 de Doutorado, 3 de Mestrado Profissionalizante (dos quais apenas 1 funciona normalmente) e 34 de Mestrado/Doutorado. Geograficamente, a expansão 
Mestres e Doutores Titulados

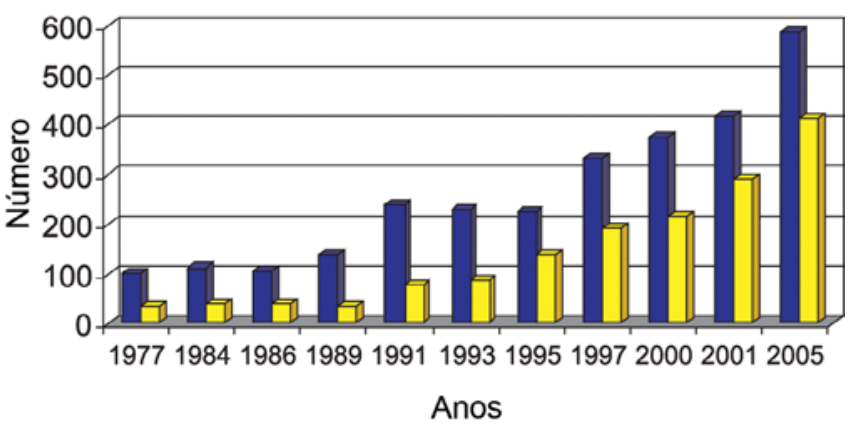

mestrado $\square$ doutorado

Figura 1. Dissertações e teses defendidas no período de 1977 a 2005

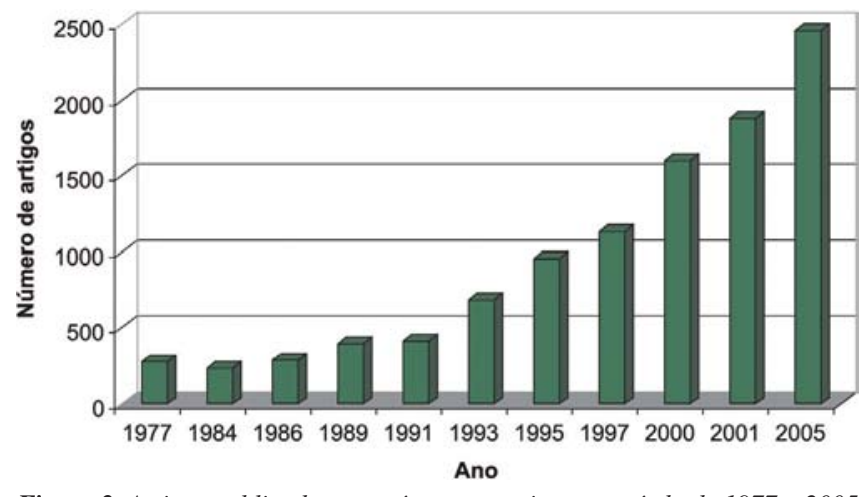

Figura 2. Artigos publicados no país e no exterior no período de 1977 a 2005

está atingindo as mais diversas regiões, de Norte a Sul do país ${ }^{21}$.

A Tabela 1 mostra a distribuição percentual dos Programas de Química em dez/2002 e em dez/2006.

Tabela 1. Distribuição Regional (\%) dos Programas de PósGraduação em Química

\begin{tabular}{cccccc}
\hline & N & NE & CO & SE & S \\
\hline $\mathbf{2 0 0 2}$ & 5 & 23 & 7 & 49 & 16 \\
$\mathbf{2 0 0 6}$ & 5 & 22 & 7 & 46 & 20 \\
\hline
\end{tabular}

Apesar do aumento no número de Programas, observa-se que a distribuição regional ficou praticamente constante. Esse aumento mostra reflexos mais evidentes sobre a demanda de alunos para os Programas da região sudeste. O número de alunos diminuiu nos últimos anos, especialmente para os Cursos de Mestrado. Para o Doutorado a demanda ainda está sendo mantida. Claro que esta constatação pode ser vista de diferentes maneiras. Pelo lado do aluno, pode ser mais interessante permanecer no seu local de origem, por uma questão de praticidade e/ou de economia. Se isso ocorre somente para o Mestrado as implicações não são tão severas. Por outro lado, a endogenia é um processo não desejável, como já foi diagnosticado no passado ${ }^{19}$. Pelo lado dos Programas, especialmente os novos, é natural que os alunos que eventualmente já estavam sendo orientados em estágios de Iniciação Científica façam parte das primeiras turmas.

Historicamente, o número de Programas sempre foi maior na região Sudeste, reflexo da distribuição de recursos dos governos esta- duais que investem em ciência e tecnologia. É também a região que abriga os maiores Programas (UNICAMP, USP/SP, UFMG e UFSCar), ressaltando-se que no Rio de Janeiro ainda predomina a existência de diversos programas divididos por sub-áreas, numa mesma Instituição. Em função desta distribuição, é ainda a região que recebe maior número de bolsas de pós-graduação, da CAPES e do CNPq, além da significativa parcela atribuída pela FAPESP e pela FAPERJ. Este conjunto de dados afeta positivamente a qualidade dos programas da região sudeste em relação às demais. Portanto, a busca de apoio das Fundações Estaduais aos Programas de Pós-Graduação é um dos fatores pelos quais a comunidade deve se empenhar.

Algumas FAP's apóiam os Programas complementando a cota de bolsas para os alunos enquanto outras apóiam projetos de pequena monta. O CNPq, via Edital Universal, também apóia projetos de médio porte. Assim, muitas vezes o esforço individual dos docentes faz com que os Programas adquiram condições para o desenvolvimento dos trabalhos de pesquisa. Alguns editais com temática específica apóiam Programas do Norte e Nordeste, principalmente, fornecendo/melhorando a infra-estrutura necessária. Por outro lado, nas demais regiões, manter e melhorar a infra-estrutura dos Programas pode ser uma tarefa bastante árdua. Muitos jovens pesquisadores estabelecem suas linhas de pesquisa graças à doação de equipamentos por parte de seus ex-orientadores. Por outro lado, falta apoio para a manutenção de equipamentos e de estrutura física dos laboratórios. Esta constatação torna-se mais séria quando o desempenho de um Programa, além da capacidade dos pesquisadores e da existência de alunos, pode ser afetado pelas condições de infra-estrutura básica. Em relação à infra-estrutura, a CAPES exige o comprometimento da Instituição, o que nem sempre acontece de forma concreta.

A meta do governo brasileiro é aumentar o número de doutores. Entretanto, esse aumento tem que garantir a manutenção da qualidade dos Programas. Para isso, é necessário estabelecer condições adequadas de infra-estrutura, diminuir a burocracia na aquisição dos reagentes e aumentar o número de bolsas em todos os Programas, novos e consolidados.

A iniciativa do Comitê da área de Química no sentido de aumentar o número de Programas é muito boa, principalmente porque está sendo feita nas diferentes regiões do país, porém, deve ser acompanhada das condições para a sua implementação. Se não há número de bolsas suficientes os alunos acabam tendo de procurar um emprego para sobreviverem e o trabalho de Dissertação/Tese passa a ficar em segundo plano. Com isso, o tempo de titulação aumenta, quando o discente consegue concluir seu trabalho. Esse fato pode desestimular o próprio docente, que investe no aluno sem a certeza de que o trabalho será concluído. Para um Programa em fase de implantação, o reflexo é ainda mais crítico.

Uma constatação emblemática em relação ao crescimento da Pós-graduação em Química é o seu corpo docente. Independentemente de todos os indicadores relativos à PG mostrarem aumentos significativos (e.g. número de alunos, número de mestres e doutores titulados, produção científica e número de cursos/programas), o número de professores atuando na pós-graduação em Química permanece estagnado desde a década de 1990 (Figura 3), mesmo considerando que no período da estagnação foram formados mais de 1400 doutores em Química no país!

Explicar a causa da estagnação do número de Docentes atuando na PG em Química não é simples, pois envolve vários fatores que vão desde a deficiência no número de novos docentes nos Institutos/Departamentos de Química, a forma de financiamento à pesquisa - que privilegia os grupos estabelecidos e promove de forma muito tímida a inclusão científica e tecnológica de novos pesquisadores o aumento do número de bolsas e oportunidades para estágios de pós-doutoramento, além da consolidação da Pós-Graduação em 
Química, cujos indicadores são cada vez mais desafiadores para os novos doutores. Reverter este quadro de estagnação é um dos desafios atuais da comunidade de química e da SBQ.

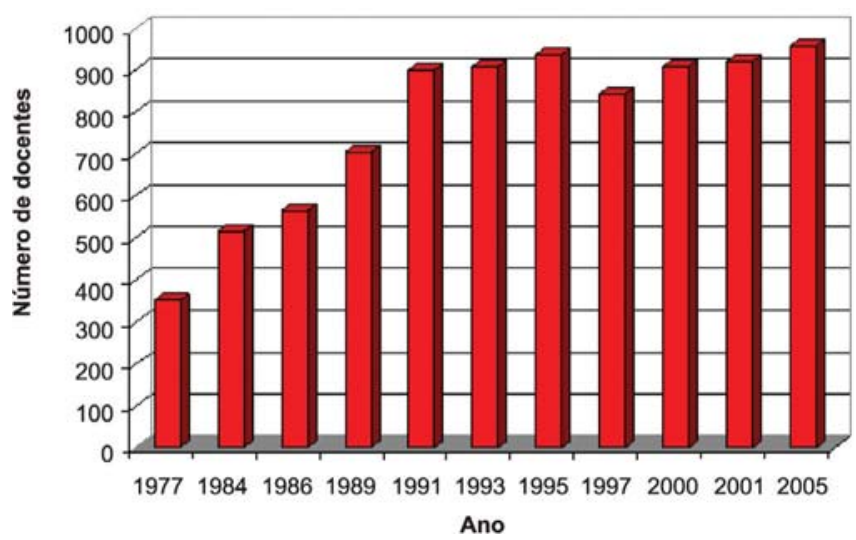

Figura 3. Número de Docentes atuando na Pós-graduação em Química no período de 1977 a 2005

Em 2005, a reunião anual dos Coordenadores de Pós-graduação converteu-se no Fórum de PG/SBQ e iniciou uma nova forma de mobilização no âmbito da Sociedade Brasileira de Química. Uma das primeiras missões do Fórum de PG/SBQ é conhecer a situação da infra-estrutura dos diversos Programas, avaliar a relação entre esse fator e o seu desempenho, verificar as necessidades mais urgentes e propor uma ação junto às agências de fomento no sentido de restabelecer as condições adequadas para o desenvolvimento da pesquisa nas diferentes Instituições. Para propor ações, foi criada uma Comissão formada pelos professores Solange Cadore (Coordenadora) (SBQ-UNICAMP), Isabel Moreira (PUC/RJ), Jari Cardoso (UFRJ), Jaísa Soares (UFPR) Maria Vargas (UFF) e Jailson de Andrade (SBQ-UFBA). No VI Workshop de Pós-Graduação, realizado em Maceió, AL, em 2006, a comissão apresentou a proposta de elaboração de um projeto mobilizador da pós-graduação em química, a ser apresentado à CAPES e aos Fundos Setoriais com o objetivo de manutenção e ampliação da infra-estrutura dos Cursos de Pós-graduação em Química, em todos os seus aspectos. Questionários para a coleta de dados já foram enviados aos coordenadores e o projeto está em fase de elaboração
Desta forma, a SBQ continua seu papel de catalisar ações para o desenvolvimento da área de Química e acompanhar o processo de ampliação, consolidação, fortalecimento e avaliação da Pós-Graduação em Química.

\section{AGRADECIMENTOS}

À SBQ pelo convite e aos Profs. A C. Pinto (UFRJ) e P. A. de P. Pereira (UFBA), pelas valiosas sugestões.

\section{REFERÊNCIAS}

1. de Andrade, J. B.; Quim. Nova 2002, 25, 891.

2. Cagnin, M. A. H.; Quim. Nova 1991, 14, 219.

3. Fatibello-Filho, O.; Neves, E. A.; Rocha, F. R. P.; Nóbrega, J. A.; Quim. Nova 2002, 25 (Supl. 1), 62.

4. Mozeto, A. A.; Jardim, W. F.; Quim. Nova 2002, 25 (Supl. 1), 7.

5. Correia, C. R. D.; Costa, P. R. R.; Ferreira, V. F.; Quim. Nova 2002, 25 (Supl.1), 82.

6. Toma, H. E.; Ferreira, A. M. C.; Serra, O. A.; Quim. Nova 2002, 25 (Supl. 1), 67.

7. Santos, P. S.; Souza, G. G. B.; Quim. Nova 2002, 25 (Supl. 1), 31.

8. Schnetzler, R. P.; Quim. Nova 2002, 25(Supl. 1), 14.

9. Oliveira, A. B.; Paniago, E. B.; Quim. Nova 1985, 8, 46.

10. Cagnin, M. A. H.; Quim. Nova 1987, 10, 223.

11. Craveiro, A. A.; Quim. Nova 1985, 8, 120.

12. Pinto, A. C.; Bechara, E. J. H.; Vichi, E. J. S.; Massaro, S.; Pardini, V. L.; Ferraz, M. H. C.; Quim. Nova 1988, 11, 167.

13. Cagnin, M. A. H.; Quim. Nova 1993, 16, 161.

14. Brocksom, T. J.; de Andrade, J. B.; Quim. Nova 1997, 20, 29.

15. da Gama, A. A. S.; Aguilera, F. N.; Machado, J. C.; Quim. Nova 1999, 22, 443.

16. da Gama, A. A. S.; Cadore, S.; Ferreira, V. F.; Quim. Nova 2003, 26, 618.

17. de Andrade, J. B.; Schor, H. H. R.; Nascimento, M. A. C.; Giordan, M.; Brocksom, T. J.; Aguilera, F. N.; Barreiro, E. J.; Quim. Nova 1995, 18, 97.

18. Sociedade Brasileira de Química; Quim. Nova 1995, 18, 509.

19. de Andrade, J. B.; Cadore, S.; Vieira, P. C.; Zucco, C.; Pinto, A. C.; Quim. Nova 2003, 26, 445.

20. de Andrade, J. B.; Cadore, S.; Vieira, P. C.; Zucco, C.; Pinto, A. C.; Quim. Nova 2004, 27, 358.

21. http://www.capes.gov.br/avaliacao/recomendados.html, acessada em Março 2007.

22. Paniago, E. B.; Quim. Nova 1997, 20 (Especial), 23.

23. Castro, L. A. B, Prescott, E.; Quim. Nova 1997, 20 (Especial), 15.

24. Braga, M. M.; Azevedo, S.; Quim. Nova 2002, 25, 696. 